\title{
Mechanically Assisted Low-Temperature Pyrolysis of Hydrocarbons
}

\author{
Aliaksandr Yurievich Alevanau1 ${ }^{*}$, Oleksandr Ivanovych Vyhoniailo², \\ Olgerd Pavlovich Kuznechik ${ }^{3}$, Pär Jönsson ${ }^{4,5}$, Mikael Ersson ${ }^{5}$, Efthymios Kantarelis ${ }^{6}$
}

\author{
${ }^{1}$ Sintos Systems Svenska Filial, Norsborg, Sweden \\ ${ }^{2}$ Ukrnaphtohimsynthes, Lisichansk, Ukraine \\ ${ }^{3}$ Observatory of Belarussian State University, Minsk, Belarus \\ ${ }^{4}$ School of Industrial Technology and Management, KTH, Stockholm, Sweden \\ ${ }^{5}$ Department of Material Science and Engineering, KTH, Stockholm, Sweden \\ ${ }^{6}$ Department of Chemistry, KTH, Stockholm, Sweden \\ Email:^alevanau@kth.se,faustus111ort@gmail.com,kuznechik@bsu.by,parj@kth.se,bergsman@kth.se,ekan@kth.se
}

How to cite this paper: Alevanau, A.Y., Vyhoniailo, O.I., Kuznechik, O.P., Jönsson, P., Ersson, M. and Kantarelis, E. (2018) Mechanically Assisted Low-Temperature Pyrolysis of Hydrocarbons. Energy and Power Engineering, 10, 133-153.

https://doi.org/10.4236/epe.2018.104010

Received: March 6, 2018

Accepted: April 8, 2018

Published: April 11, 2018

Copyright $\odot 2018$ by authors and Scientific Research Publishing Inc. This work is licensed under the Creative Commons Attribution International License (CC BY 4.0).

http://creativecommons.org/licenses/by/4.0/

\begin{abstract}
The focus of the study is experimental setups and conditions leading to pyrolysis (cracking) of such gaseous hydrocarbons as methane, mixed propane and butane, at the temperatures of the heater below $200^{\circ} \mathrm{C}$. The process was mechanically assisted by putting the substances being decomposed into a dynamic interaction with the fractal interfaces of cracks in titanium dioxide films, as well as in tin and bismuth alloy. During a trial, the alloy was periodically heated and cooled so that it changed its phase state, and fractal interfaces were created between its surface and the gases. The interaction of the gases with fractal surfaces of the alloy being produced by mechanical fracturing made it possible to obtain gas cracking even at the lower temperatures of the heater $\left(150^{\circ} \mathrm{C}\right)$. It should be noted that at this temperature, the heater couldn't melt the alloy in the heated volume with the gas.
\end{abstract}

\section{Keywords}

Pyrolysis, Porosity, Fractal Interfaces

\section{Introduction}

Technologically innovative processing of fuel types such as black oil and gaseous hydrocarbons is of valuable interest to the petrochemical industry with respect to better energy efficiency. Also, pyrolysis and refining of gaseous and liquid hydrocarbons belong to the line of socially important industries with respect to the value of their final fuel products in the forms of light hydrocarbons, hydrogen 
and carbon.

One may note that to obtain light hydrocarbons in fluidized bed reactors, the raw biomass requires an extensive mechanical processing to maximize the reactive surface of its particles and to minimize the temperature of their chemical processing [1]. In addition to this, the addition of catalytic particles to such reactors contributes to an increase of their energy efficiency by a minimization of the heat losses. These are unavoidable in high temperature applications, compared to when using catalysts.

Additions of catalysts to reactors may take place in the form of dopants of non-reactive porous particles. In such a case, an additional enhancement of the chemical reactivity takes place. This is due to the increased possibilities for reactants to reach the catalyst on the large area of the fractal surfaces in the pores. An example of an analysis of special reactive diffusion regimes on fractal catalysts is presented in [2]. Highly developed fractal geometries of internal surfaces of the pores in the particles with catalysts are beneficial for a chemical productivity and an energy efficiency of such fluidized bed reactors, as was reported in [3].

Opposite to the fractal based enhancement of the catalytic chemical mechanisms working in fluidized bed reactors, one may consider purely physical mechanisms working in reactors [4]. Accordingly to our understanding of the internal mechanisms in these reactors (where melts of non-reacting alloys of tin and bismuth are applied), it is possible to increase the rate of pyrolysis of the hydrocarbons by using dynamic interactions between the alloys and hydrocarbons on their fractal interfaces. These interfaces dynamically appear, change and disappear during motion of the hydrocarbons via the alloys. One may also create fractal interfaces mechanically at temperatures below the solidification temperature of the alloys, by using mechanical fracturing methods [5]. In the first case, the alloys are under-cooled by a convective heat transfer as is described in paper [6]. Such an under-cooling creates disordered dendritic fractal-like structures between the different phases in the alloys. Also, a dynamic solidification of these structures creates fractal interfaces between them and the cooling gas. The solidification temperature of the eutectic Sn:Bi alloy is $138^{\circ} \mathrm{C}$. The cooling gas has to have even lower temperatures. Because it appeared possible to experimentally decompose this gas during convective heat transfer from the alloy, the authors of [4] initiated a public discussion at an Internet forum (which nowadays is inaccessible) about their experimental results in 2005. The experiments showed a possibility for methane, which was chosen as a cooling gas, to be decomposed at the temperatures below $200^{\circ} \mathrm{C}$. However, accordingly to the thermodynamic calculations in [7], the minimal temperature for the start of a methane thermal decomposition is $327^{\circ} \mathrm{C}$. As it was reported on the forum, if the gas is decomposed in contact with porous silicon dioxide, or in contact with enriched metallurgical ore pellets, atomic hydrogen from the gas being decomposed is capable to reduce the oxides.

Extensive public discussions about the experiments with a substantially lower 
temperature of methane decomposition did not result in a physical explanation of these experimental results. There were no mentioning about dynamically appearing and disappearing fractal interfaces in the alloy and between the alloy and hydrocarbons during the discussions. However, the presence and possible role of these interfaces was noted by us recently due to the analysis of the papers [6] [8] [9] [10] [11] [12]. In addition to this, the emphasis on the role of fractality of the interfaces was supported by our latest experiments on the low temperature cracking of gaseous hydrocarbons in the same environment as described in the [4], but with an addition of fractal interfaces of the cracks in the titanium dioxide films. Due to all these objectivities, the current paper focuses on the physical parameters and mechanisms of the pyrolysis of gaseous hydrocarbons, which takes place due to their motion along applied fractal interfaces.

\section{Materials and Methods}

One may conclude from the introduction, that the initial working hypothesis on the physical mechanisms of pyrolysis of gaseous hydrocarbons at extremely low temperatures has its basis in the presence of fractal interfaces between the gases and the heating surfaces. Thus, the experiments were conducted with this idea in mind. To explore the effects of fractal interfaces between the heater and the gas, the following four experimental setups were made.

\subsection{The First Experimental Setup}

An under-cooling of the alloy, by using the setup in Figure 1, takes places during the motion of gas bubbles through the top part of the molten alloy in the curved glass tube. The gas inside the bubbles is initially at room temperature. Its passage via the alloy rises its temperature up to a value in the range of $95^{\circ} \mathrm{C}$ $100^{\circ} \mathrm{C}$. Such temperatures were measured using a thermocouple on the output part of a test tube with an accuracy of $\pm 1^{\circ} \mathrm{C}$. The time for a bubble to pass via the alloy is lower than one second. This time is enough for part of the gas in a bubble

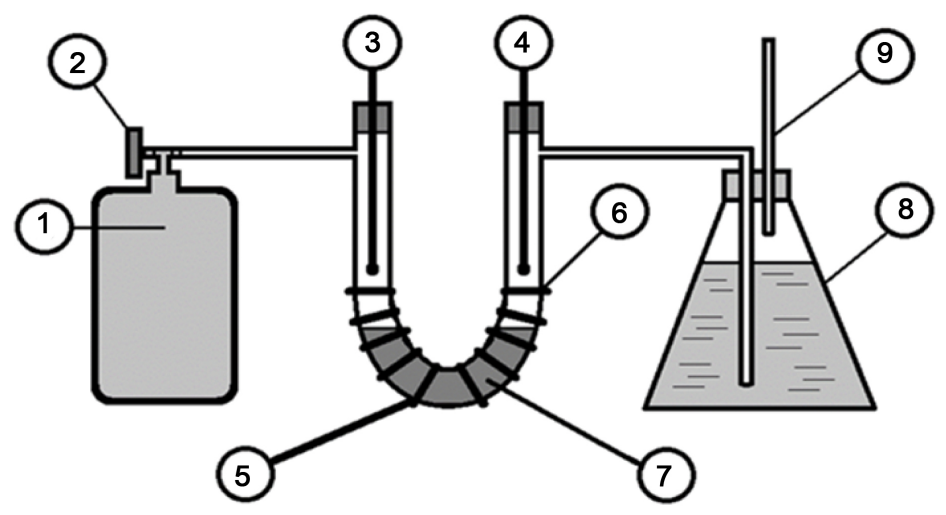

Figure 1. The first experimental setup: 1-volume containing propane/butane mixture; 2 -valve to regulate flow of gas; 3, 4, 5-thermocouples; 6-heating wire; 7-U-shape test glass with $\mathrm{Sn}: \mathrm{Bi}$ alloy; 8-volume with water; 9-output tube. 
to be decomposed and to create carbon particles. Some of these particles fall and float on the surface of the alloy forming black depositions. Photos of tops of this deposition taken after solidification of the alloy are presented in the Results section. In addition, some carbon particles get trapped inside the alloy during a passage of the bubble. These particles form porous structures inside the alloy, which presumably include hydrogen as a secondary product of hydrocarbons' decomposition upon solidification of the whole alloy volume.

\subsection{The Second Experimental Setup}

The experimental setup in Figure 2 partially reproduces the setup, which was discussed publicly on Internet by the authors of [4]. It differs by the addition of a circular loop for the gas, which after an initial filling of the tubes and test bulb is pumped by a membrane pump in a loop. A magnet (8) was used to mix the gas and alloy to obtain a better under-cooling of the alloy to produce as much carbon particles and hydrogen as possible. Because of the use of a cooler, which was made of approximately a $3 \mathrm{~m}$ copper tube submerged in a volume with cold water, the alloy was under-cooled too efficiently. It was only $15 \mathrm{~g}$ of the alloy in the bulb. Due to this, the rotation of the magnet was stopped and the alloy was periodically melted and solidified due to a periodic switching of the pump in an off and on mode. The measured temperature of the gas in the glass bubble was $90^{\circ} \mathrm{C}$, while the alloy was in a molten stage. The pump was switched off when the temperature of the gas was decreased to a temperature of $85^{\circ} \mathrm{C}$ and the alloy

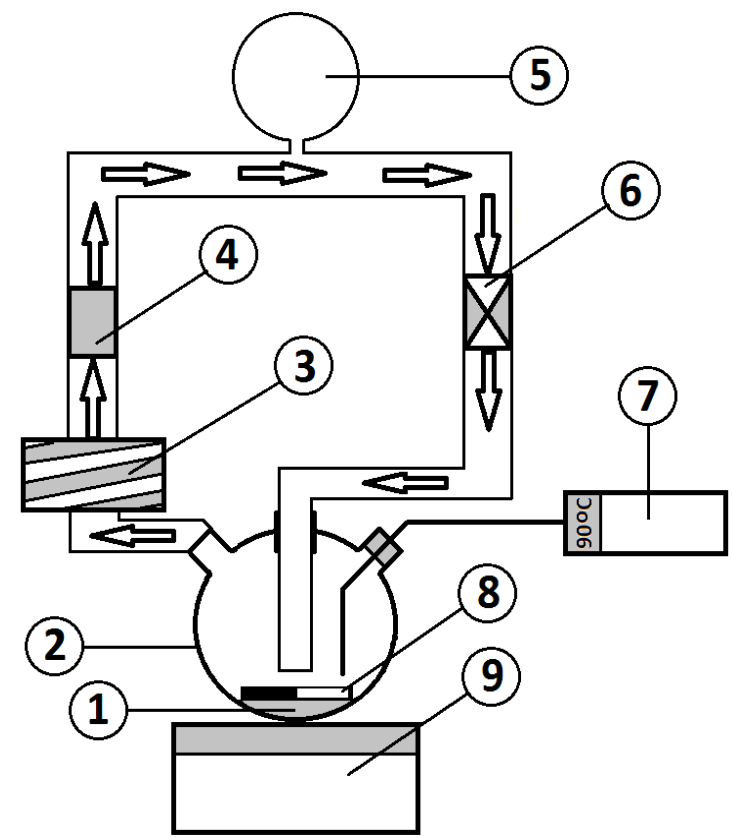

Figure 2. The second experimental setup: 1-Sn:Bi alloy; 2-glass bulb; 3-cooler; 4-filter; 5-intermediate flexible volume; 6-membrane pump; 7-thermometer with K-type thermocouple attached; 8-magnet in a plastic cover; 9-thermo-stabilized heater with magnetic mixer. 
became solid. It is necessary to note, that the end of the thermocouple was not submerged in the alloy. Instead the temperature was measured at a small distance from its surface. Because of trapping of carbon particles to the alloy being solidified, its melting took place slower than it could have proceed without the presence of particles. Due to the strong pulsating blowing of the gas being pumped by the membrane pump, the boundary part of the alloy often became separated from its main part up to the end of blowing cycle. The maximum temperature of the heater was $250^{\circ} \mathrm{C}$. Also, the experiments included measurements of the gas composition by using Agilent Technologies 490 Micro GC equipment.

An important note was made during observation of the dynamics in the bulb before a melting of the alloy took place. This note is described in more detail below. It yielded a modification of the experimental setup, which is described in Figure 3.

The alloy was prepared in the form of chopped sticks of a wire with a diameter $2.5 \mathrm{~mm}$. The sticks were chopped in such a way, that their ends were stretched and broken during the process. In such a case, the surface of a broken alloy acquires a fractal-like geometry [5].

The sticks were mixed by a magnet during the initial step-by-step heating of a bulb. The gas was also pumped during this heating. It was noted during the observations of this process that tiny black bars appeared among the alloy sticks. The length of the bars was comparable with the diameter of the chopped wire sticks of the alloy. These tiny black bars disappeared when the motion of a magnet

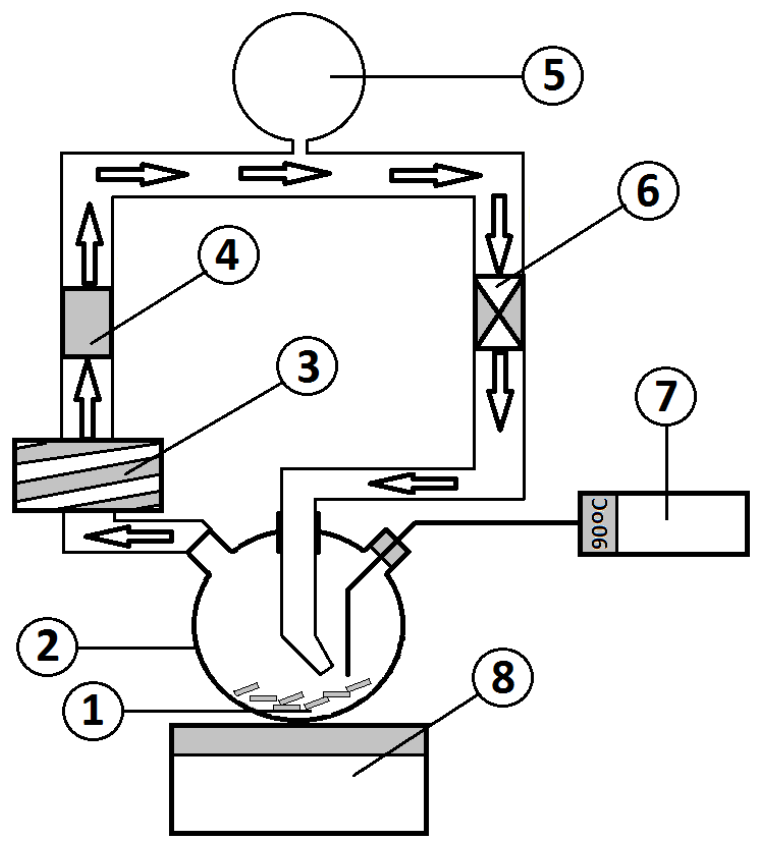

Figure 3. The third experimental setup: 1-Sn:Bi alloy sticks; 2-glass bulb; 3-cooler; 4-filter; 5-intermediate flexible volume; 6-membrane pump; 7-thermometer with K-type thermocouple attached; 8-thermo-stabilized heater. 
and pumping of the gas was switched off and a melting of the alloy was achieved. However, their appearance was noted and hypothetically linked to the existence of fractal surfaces on the ends of the chopped alloy sticks.

\subsection{The Third Experimental Setup}

To test the hypothesis about a decomposition of butane/propane mixture due to its motion along the fractal surfaces on the ends of the sticks, the experimental setup illustrated in Figure 3 was made. The mixing of the sticks by a magnet and their melting were not assumed during the experiments using this setup.

These experiments were conducted in a glass bulb at a $150^{\circ} \mathrm{C}$ temperature of the heater, and with a maximum temperature of $70^{\circ} \mathrm{C}$ of the gas. It was observed that some of the alloy sticks were constantly moving and colliding under the pulsating blowing of the gas, coming from the membrane pump.

\subsection{The Fourth Experimental Setup}

The fourth experimental setup is the same as the second setup except for an addition of an electrochemically processed titanium tape being placed in the close proximity to the surface of the molten alloy. The tape receives a relatively thick layer of a titanium dioxide after its processing by electrolysis in a water solution of barium hydroxide. The tape is applied as a positive electrode, which is being placed in the form of a cylindrical spiral into a closed plastic tube. The tube has a diameter of $30 \mathrm{~mm}$ and a height of $60 \mathrm{~mm}$. A second titanium electrode is mounted on the lid of the tube as a bar with the diameter $4 \mathrm{~mm}$. It stretches down along the axis of a cylindrical spiral. A rectified current for electrolysis is supplied accordingly to the parameters described in the patent SU1838455A3. The use of those parameters resulted in a thickness of the titanium oxide films in the range of $10-15 \mu \mathrm{m}$.

The main property of the oxide films being obtained by electrolysis in such a geometry is presence of many holes in the films. These holes are created by the current that is generated between the electrodes. Due to presence of a curvature of the electrode in the form of a cylindrical spiral, the holes in the film on it become cracks when the tape is extracted from the tube and is straightened. Even more cracks appear in the oxide film if the tape is bent into the opposite direction with respect to its geometry during the electrolysis. This is a well-known fact that internal surfaces of such cracks are fractals. Thus, this is the third way to obtain a fractal geometry on the interfaces between the hot solids and cold gases, whose low-temperature decomposition is in the focus of this work.

\subsection{Generalities in All the Experimental Setups}

Due to the highly dynamic character of the heat transfer processes, which were applied in all the experiments, the K-type of thermocouples was the optimal type in regard to their inertial response in the intruder method of the temperature measurements. As the start-stop of blowing of the cooling gas was governed 
mostly by visual perception of solidification of the alloy, exact temperature readings to control the process were unnecessary. Due to the fact, that all the experimental setups were oriented on the detection of the process of low temperature hydrocarbons' decomposition, it was not possible to predict characteristics of the carbon particles, which could agglomerate differently in different environments and conditions. Thus the particles are described in results of the experiments only by their perception by naked eye, and by optic microscopy. The gas chromatograph also was applied for detection of the fact of hydrocarbons decomposition using observation of the hydrogen content in the gas.

Another method to detect even atomic hydrogen from the hydrocarbons was applied in the last experimental setup. This method assumes reduction of titanium oxides by the atomic hydrogen, which is assumed to be extremely reactive inside the fractal fractures of the oxides.

One may evaluate efficiency of all the applied methods in the next sections describing experimental results of every experimental setup.

\section{Results}

\subsection{Results of the Experiments Using the First Experimental Setup}

The experiments using setup in Figure 1 were conducted using two different alloys. The first one has a $57 \% \mathrm{Sn}$ and $43 \%$ Bi composition with an eutectic temperature of $138^{\circ} \mathrm{C}$. The second alloy consisted of $54 \% \mathrm{~Pb}, 11 \% \mathrm{Sn}$ and $35 \% \mathrm{Bi}$. It has a $140^{\circ} \mathrm{C}$ melting temperature. Accordingly to [13], inclusions of lead in the alloy results in much more complicated structures of different phases in different conditions in comparison to a eutectic Sn:Bi alloy. However, the alloy with lead showed a lower efficiency in comparison to the eutectic Sn:Bi alloy with respect to the production of both carbon particles and hydrogen from the propane/butane mixture source. One may see the difference with respect to density of the particles trapped on the surfaces of the alloys in Figure 4 and Figure 5. The measured maximum and minimum contents of hydrogen in the gas were $158.7 \mathrm{ppm}$ and $60 \mathrm{ppm}$, respectively for both alloys. Small amounts of intermediate products of propane/butane decomposition such as ethylene and propylene were also present in the gas. As the hydrogen content was approximately three times higher in the experiments with an eutectic Sn:Bi alloy compared to the experiments with a $\mathrm{Sn}: \mathrm{Bi}: \mathrm{Pb}$ alloy, all the subsequent experiments were conducted with the Sn:Bi alloy only.

Because of trapping of carbon particles and hydrogen inside the alloy during its solidification, a slow pressure drop was observed in the tube between the test glass and a barrier volume containing water. Water from this volume was sucked into the alloy during 8 hours of cooling of all the parts in the setup to room temperature. This is due to a diffusion of hydrogen out from the porous structure in the solidified alloy. Furthermore, by its replacement with the propane/butane mixture resting in the tube. In the case of a tight tube connection 


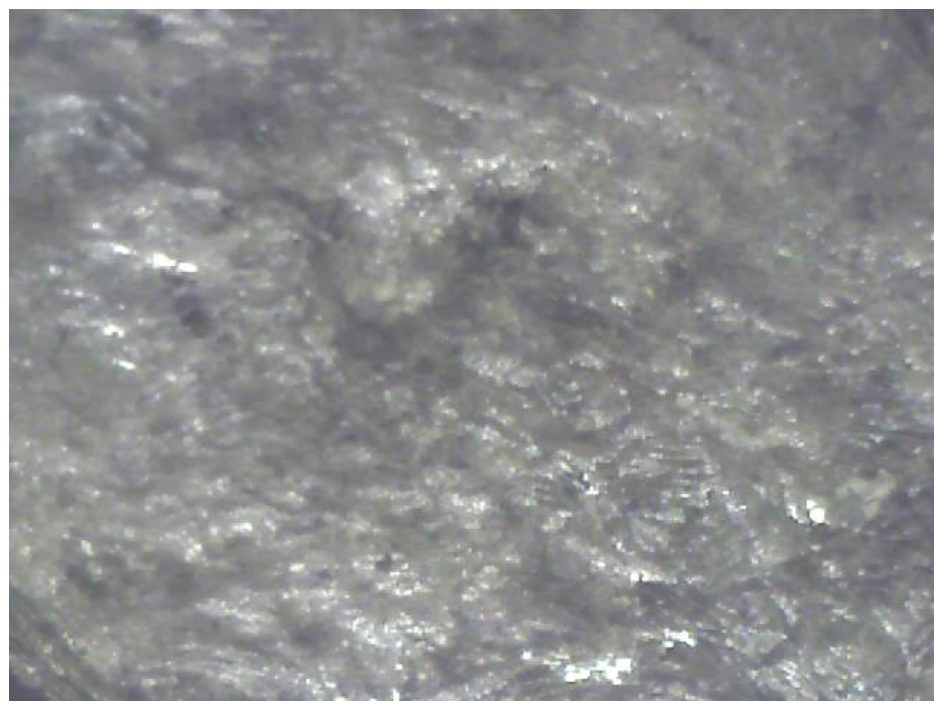

Figure 4. Carbon particles on the surface of $\mathrm{Sn}: \mathrm{Bi}: \mathrm{Pb}$ alloy (app. 150x optical magnification).

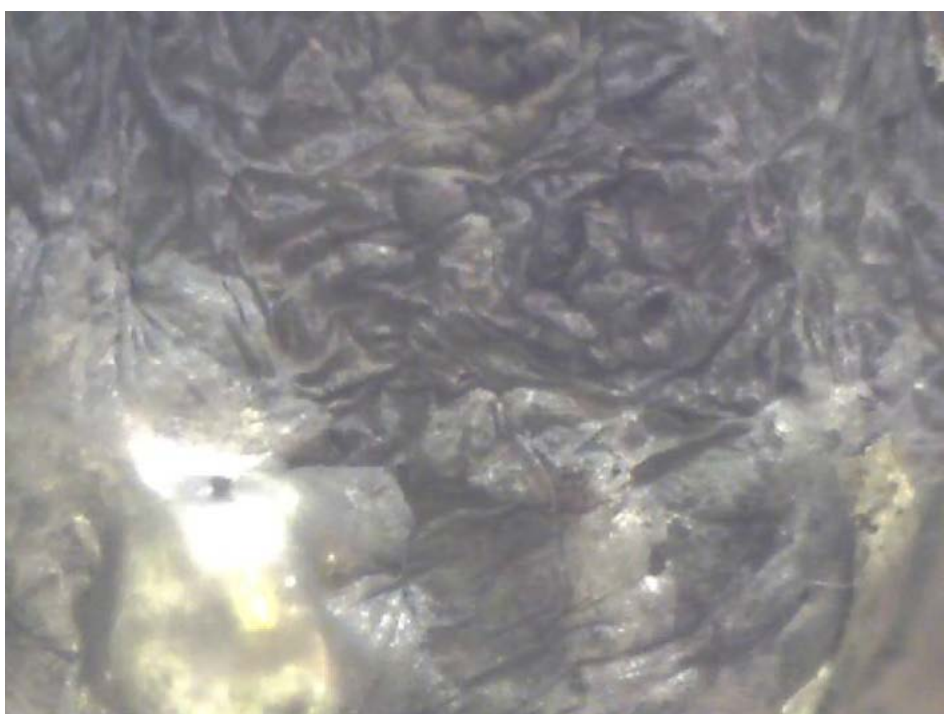

Figure 5. Carbon particles on the surface of Sn:Bi alloy (app. 150× optical magnification).

between the test glass and a barrier volume with water, the water may come in a contact with the alloy due to a pressure drop in the tube.

\subsection{Results of the Experiments Using the Second Experimental Setup}

Carbon particles are produced during the periodic blowing, when the pump is switched on and off. Figure 6 shows a photo of the filter before and after an experiment using the setup No. 2 described in Figure 2. During the experiment the particles and propane/butane mixture were moving in a loop above the alloy. Also the alloy was under-cooled by this motion, and it melted when this motions was stopped. 


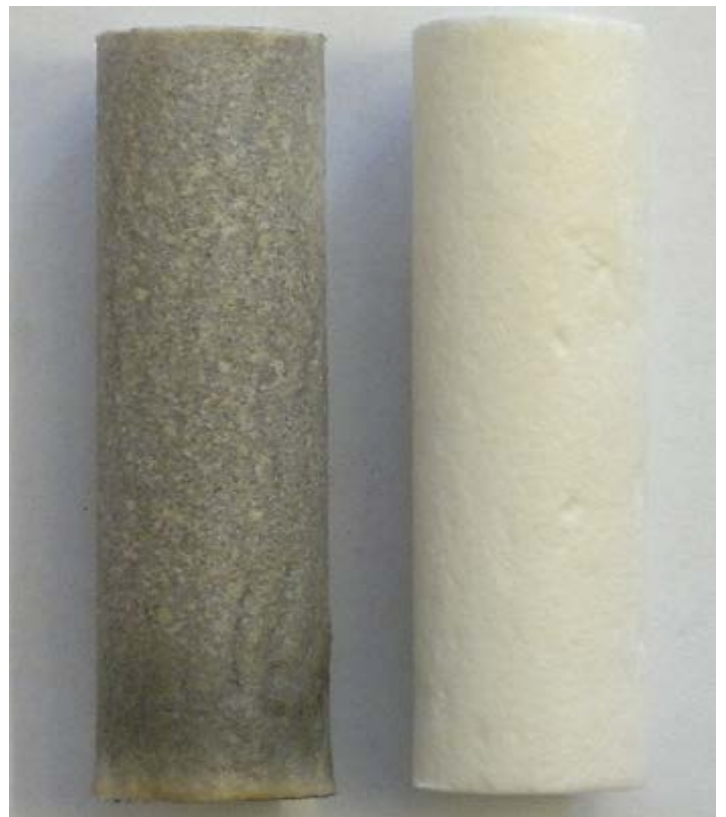

Figure 6. Filter with and without carbon particles.

The particles were also observed in the flexible intermediate volume, where agglomerations in the forms of triangles were found. These agglomerations were easily moved (they were jumping) by static electricity from fingers while touching the surface of a partially transparent volume.

The gas, which was collected in the intermediate volume along with the particles, was injected to the Agilent Micro GC at the end of an experiment. The GC measurements showed a maximum percentage of the hydrogen content $(0.1 \%)$ among the other hydrocarbons in the mixture. Previous injections showed varying compositions of the mixture, which was originated from the source propane/butane mixture.

The composition of the initial propane/butane mixture: $\mathrm{C}_{3} \mathrm{H}_{8} \quad 16.78 \%, \mathrm{iC}_{4} \mathrm{H}_{10}$ $18.22 \%, \mathrm{nC}_{4} \mathrm{H}_{10} 65.0 \%$. The most probable reasons for the variations are the periodic start-stop regime of the flow and the pretty big volume of the gas in the loop. The results of four short-time characteristic series of measurements are presented in Table 1.

The carbon particles were also deposited on the surface of a magnet, which was applied for mixing of chopped sticks of the alloy before it was melted. At the same time an appearance of the tiny black bars was noticed. They were mixed by a magnet together with the sticks in the glass bulb. When the alloy was melted, these bars disappeared in the melt.

Their expected appearance took place during the experiment using setup No 3, which is shown in Figure 3.

\subsection{Results of the Experiments Using the Third Experimental Setup}

The photo of the bars, which look like foils detached from the ends of the sticks 
Table 1. Gas composition measurements in the experiments using setup No 2.

\begin{tabular}{ccccccccccc}
\hline \multirow{2}{*}{$\begin{array}{c}\text { Time } \\
(\text { min })\end{array}$} & \multicolumn{10}{c}{$\mathrm{Mol}(\%)$} \\
\hline 0 & $\mathrm{H}_{2}$ & $\mathrm{CH}_{4}$ & $\mathrm{CO}$ & $\mathrm{CO}_{2}$ & $\mathrm{C}_{2} \mathrm{H}_{6}$ & $\mathrm{C}_{3} \mathrm{H}_{8}$ & $\mathrm{C}_{3} \mathrm{H}_{6}$ & $\mathrm{C}_{4} \mathrm{H}_{10}$ & $\mathrm{C}_{3} \mathrm{H}_{4}$ & $\mathrm{CH}_{3} \mathrm{C}_{2} \mathrm{H}$ \\
\hline 8 & 0 & 0 & 0 & 0.035 & 0.194 & 27.068 & 40.503 & 6.792 & 25.408 & 0 \\
27 & 0.009 & 0 & 0 & 0.046 & 0.393 & 36.571 & 16.360 & 14.465 & 32.153 & 0.003 \\
30 & 0 & 0 & 0 & 0.050 & 0.421 & 30.925 & 21.299 & 15.215 & 32.086 & 0.003 \\
36 & 0.006 & 0 & 0 & 0.049 & 0.427 & 54.358 & 8.170 & 14.392 & 22.596 & 0.002 \\
39 & 0.057 & 0 & 0 & 0.052 & 0.448 & 56.545 & 8.079 & 14.679 & 20.137 & 0.003 \\
88 & 0.001 & 0.122 & 5.300 & 0.024 & 0.184 & 34.964 & 39.783 & 6.704 & 12.919 & 0 \\
92 & 0.103 & 0 & 2.692 & 0.133 & 0.796 & 1.385 & 59.942 & 21.623 & 13.325 & 0 \\
95 & 0.030 & 0 & 0 & 1.077 & 0.539 & 69.629 & 12.268 & 16.457 & 0 & 0 \\
\hline
\end{tabular}

during their collisional mixing, is presented in Figure 7. These bars contain carbon depositions determined using SEM, which are more extensive in comparison to the depositions on the fractal surfaces on the ends of the alloy sticks (see Figures 8-10). Because of this, the bars looked black under the observation by a naked eye. Also, their lengths were comparable with the diameter of the sticks, see Figure 10. However, some of them could form bars of a double the length by electrostatic sticking one to another (see Figure 7).

Measurements using energy dispersive spectroscopy (EDS) at the electron microscope showed the following mass percentage of the elements in the surface of a tiny bar near the end of an alloy stick in Figure 10: 37.8\% C, 27.8\% Sn, $34.3 \% \mathrm{Bi}$. As the surface of the bar looks black, one may assume that a deposition of carbon particles on it is pretty dense. This is supported by the atomic ratios given by the EDS measurements: $88.76 \%$ C, $6.60 \%$ Sn, $4.64 \%$ Bi. However, these values are not the same for every chosen bar with such a deposition. Some EDS measurements could also show a small presence of $\mathrm{Fe}$ on the bars. This element might appear on the bars from the cutters applied to cut the alloy sticks.

Opposite to the results of the experiment using setup No 2, no visible depositions of the carbon particles appeared on the filter in the loop during approximately the same experimental time in the experiments using setup No 3.

The same note applies for the experiments using setup No 3, where flexible volume with the propane/butane mixture was replaced by the volume with biogas from Stockholm Vatten AB production facilities. This biogas had a $66.36 \%$ methane and a $33.63 \%$ carbon dioxide composition. Even if the percentage of the hydrocarbon in this mixture is not large, the noticeable amounts of hydrogen were measured during the experiments and using continuous and start-stop blowing regimes of the alloy application. In this experiment, the temperature of molten alloy didn't exceed $170^{\circ} \mathrm{C}$. The results of the gas measurements are presented in Table 2. 


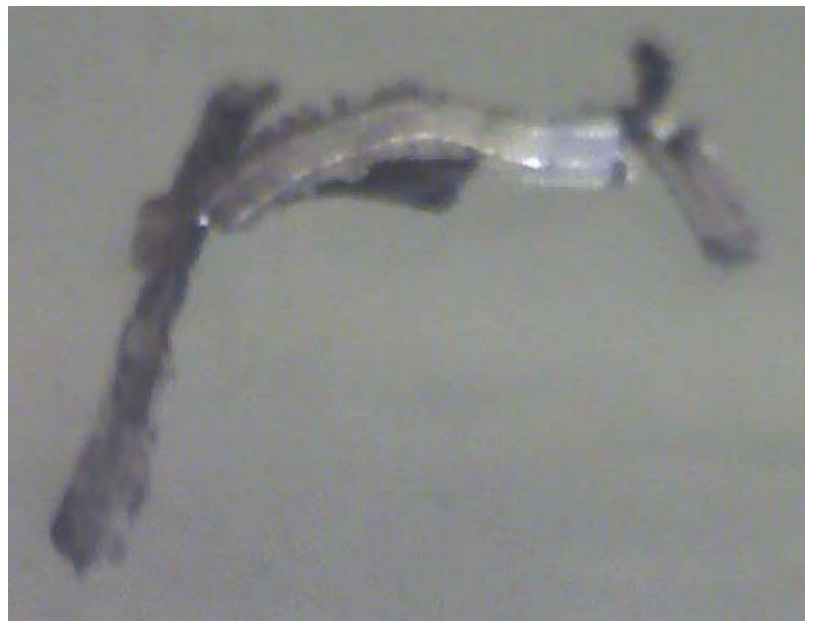

Figure 7. Foils detached from the alloy sticks.

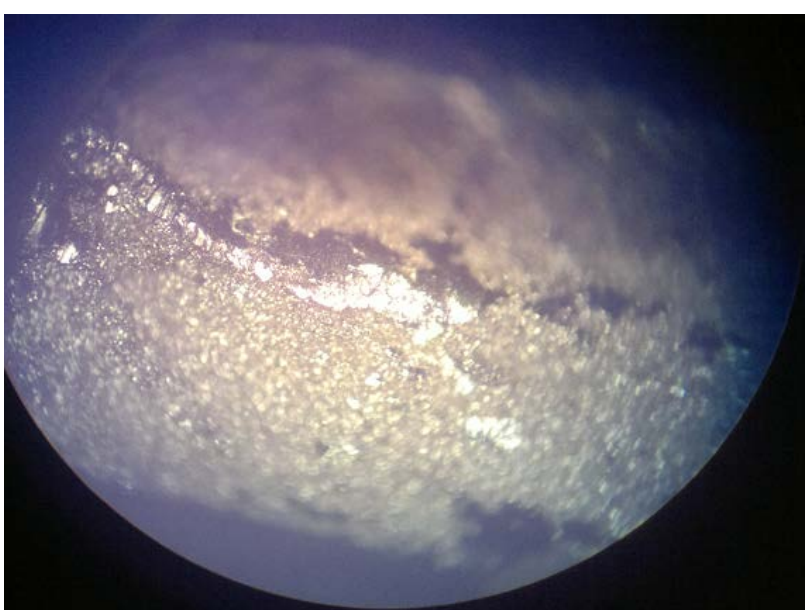

Figure 8. Black deposition on the end of a chopped alloy stick (80× optical magnification).

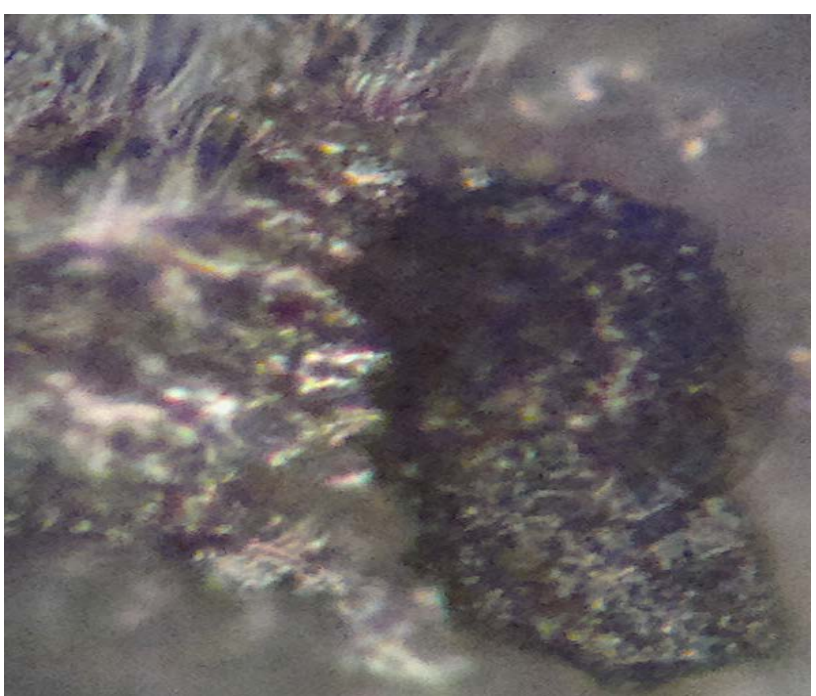

Figure 9. Black deposition on the fractal surface of a chopped alloy stick (500× optical magnification). 


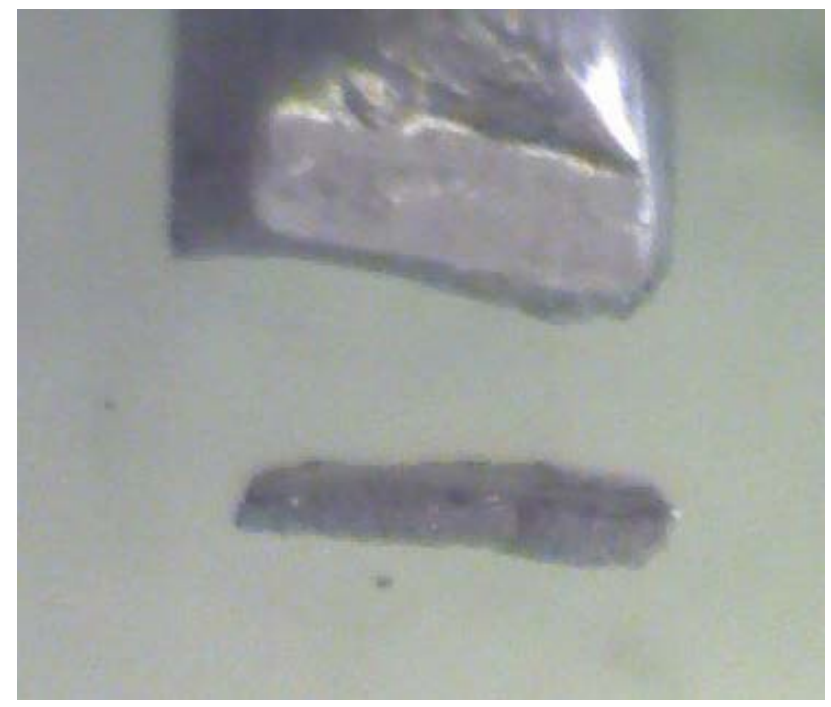

Figure 10. Black bar (foil) near the end of a chopped alloy stick.

Table 2. Biogas composition results from measurements in the experiment using setup No 2 .

\begin{tabular}{ccccc}
\hline \multirow{2}{*}{$\begin{array}{r}\text { Time } \\
(\mathrm{min})\end{array}$} & $\mathrm{H}_{2}$ & $\mathrm{CH}_{4}$ & $\mathrm{CO}$ & $\mathrm{CO}_{2}$ \\
\cline { 2 - 5 } & 0.004192 & 66.08018 & 0.030457 & 33.11823 \\
0 & 0.002843 & 65.86591 & 0.011658 & 34.11959 \\
3 & 0.004289 & 65.84567 & 0.022134 & 34.1279 \\
7 & 0.004225 & 65.83151 & 0.01487 & 34.14939 \\
10 & 0.003035 & 65.7556 & 0.0 & 34.24137 \\
16 & 0.0038 & 65.98002 & 0.005353 & 34.01083 \\
\hline 19 & & & & \\
\hline
\end{tabular}

\subsection{Results of the Experiments Using the Fourth Experimental Setup}

The most impressive result of these experiments was found by a direct observation of the low electrical resistance (below $100 \mathrm{Ohms}$ ) between the random spots on the titanium dioxide film at the titanium tape, which was bent in an opposite direction in comparison to its original curvature during an electrolysis. The same experiments with the only straightened titanium tapes couldn't show any electrical resistance of the random spots on the tapes. Resistance measurements were conducted after processing of the tapes in an experimental environment of setup No 2. They were made by an ordinary ohmmeter having its upper measurable resistance limit a one hundred mega ohms.

The obvious conclusion about bigger cracks to be produced in the titanium dioxide films on the tapes in the case of their stronger bending raised a question about the origin of the cracks' conductivity. The cracks, which are visible under an optical microscope, are too small $(<10 \mu \mathrm{m})$ and rare to provide a direct contact with a titanium tape below its oxidized surface, which is pretty thick and 
solid.

Conductivity via the cracks may appear in the case of their filling by a conducting material and in the case of acquiring of conductivity by the cracks' walls. Both cases may work in the case of hydrocarbon decompositions inside the cracks. In such case, a filling of the cracks by the carbon particles being obtained from the hydrocarbons is possible. Moreover, a titanium dioxide reduction by the atomic hydrogen being produced inside the cracks, from the same hydrocarbons, is also possible.

An externally originated filling of the cracks by current conducting particles, which fly from the surface of the phase changing alloy, is also possible. The phase separation on the surface of the under-cooled alloy in conjunction with a strong gas blowing may produce small metallic particles that follow the flow of a gas and fill the cracks in the closely located oxidized tapes.

Measurements of elemental composition by the EDS method being applied for a large surface area of the tape showed notable differences (30\% and 50\% respectively) of ratios between the titanium and oxygen on the surface, and in a deep spot inside a typical conductive crack. These measurements also confirmed the possibility for small metallic particles from the alloy to be detached from its surface, and be trapped inside the cracks on the tape surface. In particular, EDS measurements in point 3 inside the crack being presented at Figure 11 showed the presence of $\mathrm{Bi}$ in the point.

Figure 12 shows a surface of the Sample 1, which wasn't bent into the opposite direction in comparison to its geometry during electrolysis. Both samples 1 and 2 were taken from adjacent parts of the tape. EDS measurement taken at a relatively large rectangular area of Sample 1 shows nearly the same values

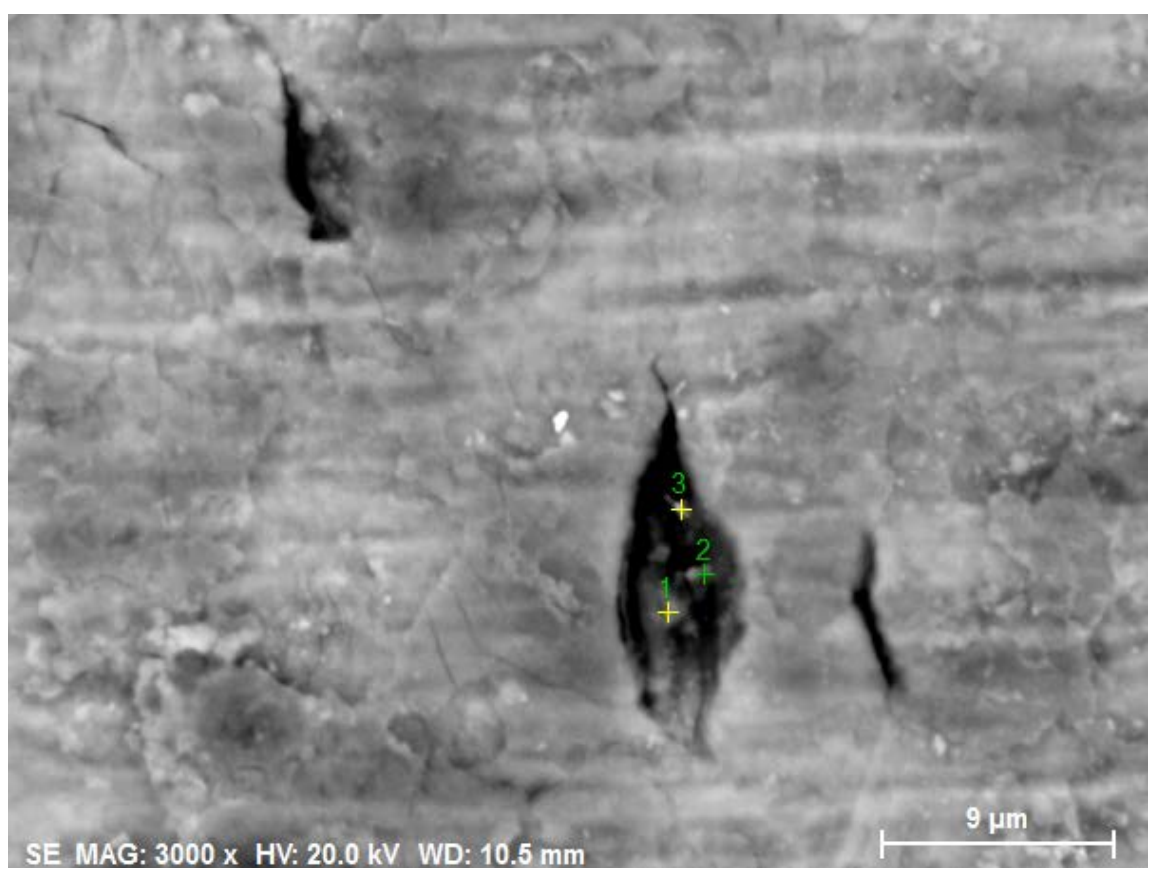

Figure 11. EDS points in the crack on Sample 2. 


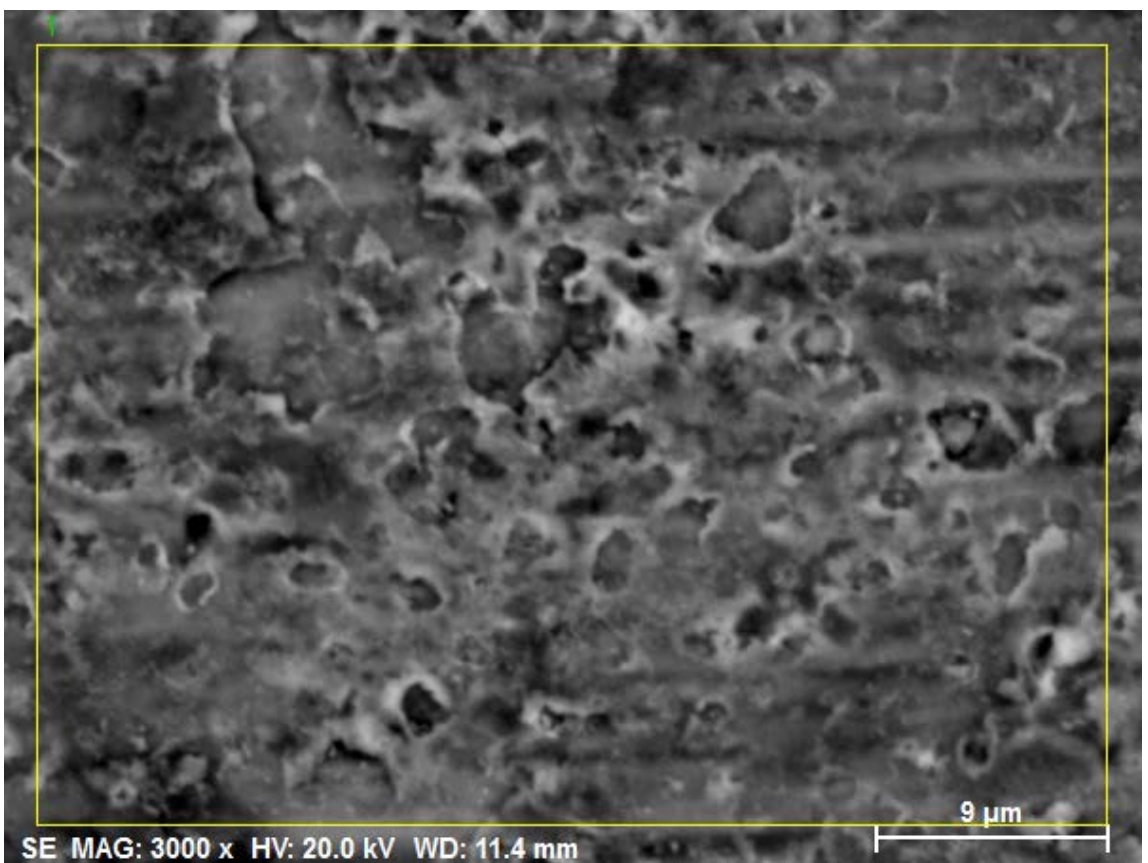

Figure 12. EDS area of the sample 1.

( $13.79 \%$ vs $13.42 \%$ and $15.92 \%, 62.76 \%$ vs $61.23 \%$ and $60.23 \%, 23.45 \%$ vs $25.35 \%$ and $23.46 \%$ ) of the barium, titanium and oxygen contents, as they are in the points 1 and 2 of the crack from Sample 2. The point 3, which is deeper in the crack, contains no barium, but bismuth (8.29\%) and sodium (0.37\%). However, it has a reduced percentage of oxygen (11.84\%) along with an increased percentage of titanium (79.50\%). Even if EDS measurements may not be applied for an exact quantification of carbon element, the amplitude of its peak at the spectrum from the rectangular surface in Sample 1 is two times lower in comparison to the amplitude on the spectrum taken in point 3 inside the crack of Sample 2. This may indicate the above mentioned reduction of titanium dioxide by the hydrogen from the hydrocarbons, even if such reactions are prohibited by thermodynamics at the temperatures of the conducted experiments (around $140^{\circ} \mathrm{C}$ ).

The data from EDS measurements are presented in Table 3. Here the notion Sample 1.1 designates a rectangular EDS measurement area of the Sample 1, the Sample 2.1 the EDS measurement point 1 inside the crack of Sample 2, etc.

\section{Discussion}

The most valuable result of the experiments using a pulsating flow of the gas and the rapidly changing gradients of temperature on the heat conducting fractal interfaces is the confirmation of a special role of these interfaces in the process of a gas decomposition.

The appearance of carbon particles in the case of interaction of hydrocarbons with mechanically created fractal interfaces being subjected to the pulsating blowing by a cooling gas provides more support for the hypothesis, which was formulated by a correspondent author of this paper in [14]. This hypothesis 
Table 3. Results of EDS measurements for two samples.

\begin{tabular}{ccccc}
\hline \multirow{2}{*}{ Element } & \multicolumn{4}{c}{ Wt (\%) } \\
\cline { 2 - 5 } & Sample 1.1 & Sample 2.1 & Sample 2.2 & Sample 2.3 \\
\hline Oxygen & 23.45 & 23.35 & 23.46 & 11.84 \\
Titanium & 62.76 & 61.23 & 60.63 & 79.50 \\
Barium & 13.79 & 13.42 & 15.92 & \\
Bismuth & & & & 8.29 \\
Sodium & & & 0.37 \\
\hline
\end{tabular}

explains the decrease of the activation energy of pyrolysis reactions in samples of wood and other biomasses, which have extensive internal gradients of temperature and pressure. The decrease of the activation energy is analyzed in [14] from the point of view of a fundamental fractal theory [10] [11] [12]. Its explanation using the theory provides an illustrative support for an understanding of the experimental results reported in this paper. Fractal surfaces of pores in the wood samples, which have an extensive transport of the phase changing hydrocarbons moving in the pores due to the dynamic gradients of pressure and temperature, are analogous to the fractal surfaces of under-cooled $\mathrm{Sn}$ :Bi alloys, or to fractal surfaces in the titanium dioxide cracks. Furthermore, to the fractal surfaces on the ends of the chopped alloy sticks. The dynamic gradients of pressure and temperature with respect to the experimental conditions applied in the described experiments are created by the rapid changes of the temperature and pressure in the proximity to these heat conducting fractal interfaces.

These conditions are necessary for a nontrivial Laplacian in the description of the heat diffusion process according to paper [11]. Specifically, Equation (1) for the generalized quantum force $Q$ can be used in the system with the mass $m$ in the dynamic conditions of energy and mass transfer driven by the potential $P$ :

$$
\frac{Q}{m}=-2 D^{2} \nabla\left[\frac{\Delta \sqrt{P}}{\sqrt{P}}\right]
$$

where the constant $D$ is assumed to be proportional to the diffusion coefficient in the equation (which is not included here) describing the dynamic heat or mass transfer driven by the potential $P$. This transfer spatially includes an oscillator, which obtains an additional energy due to the dynamic character of the transfer, and due to the hypothetical fractality of the space-time.

One may consider hydrogen-carbon bonds in molecules of hydrocarbons as approximate examples of harmonic oscillators. If those oscillations are described by a standard equation $x=a \cdot \cos (w t)$, one can calculate the additional energy $(D w)$ to be gained by such oscillators accordingly to the Equation (2) cited from [12]:

$$
E=D w+\frac{1}{2} a^{2} w^{2}
$$

where $w$ is the frequency and $a$ is the amplitude of oscillations. 
The consideration in [12] was made for the case with the absence of an explicitly presented fractal geometry, which may change the dynamics of the oscillator. To show the presence of this geometry in the geodesic motions of space-time, one needs to apply a special retro-active force loop to the oscillator. The purpose of this retro-active force loop is to tune the form of oscillations to the form predicted by the theory [10], for oscillations of a generalized quantum wave packet.

According to our understanding of the basic concepts of the theory [10], such an artificial tuning is unnecessary in the case of a natural fractal geometry being present along with the presence of dynamic potentials driving the transfer processes. The latter involve oscillators of different sizes and scales. In such a case, the oscillators may efficiently and indirectly obtain an additional energy for their oscillations due to appearance of generalized quantum potentials. These may have their origin at the presence of dynamic potential gradients, fractal properties of space-time, and fractal properties of experimental environments and conditions.

It also looks possible to interpret such an indirect energy transfer to the oscillators as a direct manifestation of a Le Chatelier principle. In this context, the system in the form of a tiny boundary layer mediating convective heat transfer will tend to preserve its equilibrium state against spreading of a sharp rise of the temperature. Accordingly to the Notalle's theory [11] and the Equation (1), such a preservation is to be accomplished by acquiring molecules of hydrocarbons of stochastically notable impulses from the quantum-like force $Q / m$ deviating their oscillations to the same direction against the direction of the sharp temperature front spreading.

Taking this consideration into account, one may conclude that intermolecular vibrations in hydrocarbons will efficiently obtain this indirectly transferred energy during their passage and the convective heat transfer along the geometries of the hot fractal interfaces.

Thus, the probability of the subsequent hydrocarbons' decomposition due to a collisional energy exchange between their molecules will be increased. This may explain the appearance of carbon particles and their agglomerations after the passage of the propane/butane gas mixture via the filter in the loop.

Another explanation for hydrocarbons' decompositions is in catalytic mechanisms, whose action is to be amplified by the fractality of the surfaces interacting with hydrocarbons. Because the passage of the gas being decomposed via the filter, and because the subsequent agglomeration of the carbon particles takes place in a flexible volume, this explanation seems to be applicable only in the case if the small particles of the alloy promote hydrocarbons' decompositions. Furthermore, if they are moved with the gas being small enough to pass via the filter. The EDS measurements showed a presence of bismuth (8.29\%) inside the titanium dioxide cracks. This may happen only in the case of a separation of bismuth and tin phases during a dynamical surface solidification of the 
alloy, due to its convective cooling. The smallest parts of the dynamically forming fractal interfaces between the phases of the alloy may become mechanically detached from the surface by a strongly blowing gas. Thereby, they will be transferred by the gas flow in the loop and promote a catalytic gas decomposition.

There is also an argument against the note about catalytic action of small particles of the alloy moving with the cooling gas. This argument comes from an industrial practice of the authors of [4]. They do not apply the phase changing alloy in a direct contact with the hydrocarbons being processed. The alloy dynamically changes its phases being hermetically welded inside the steel plates. The plates cover the chambers with heated biomass. Thereafter, they conduct the heat, which also passes through the alloy. Because an active mixing of biomass in a close contact with the plates, the alloy dynamically changes its phase inside the welded steel plates.

An example of such reactor is presented on Figure 13. This reactor moves wood chips and other biomass from harvest rests by an Archimedes screw rotating inside the chamber, which is heated through the alloy being hermetically welded between the steel plates. As it is reported by one of co-authors of this work, exploitation of this reactor showed economical feasibility of such its construction and application of the alloy as an intermediate material conducting the heat to biomass. The active coal, which is produced by this reactor, has a good

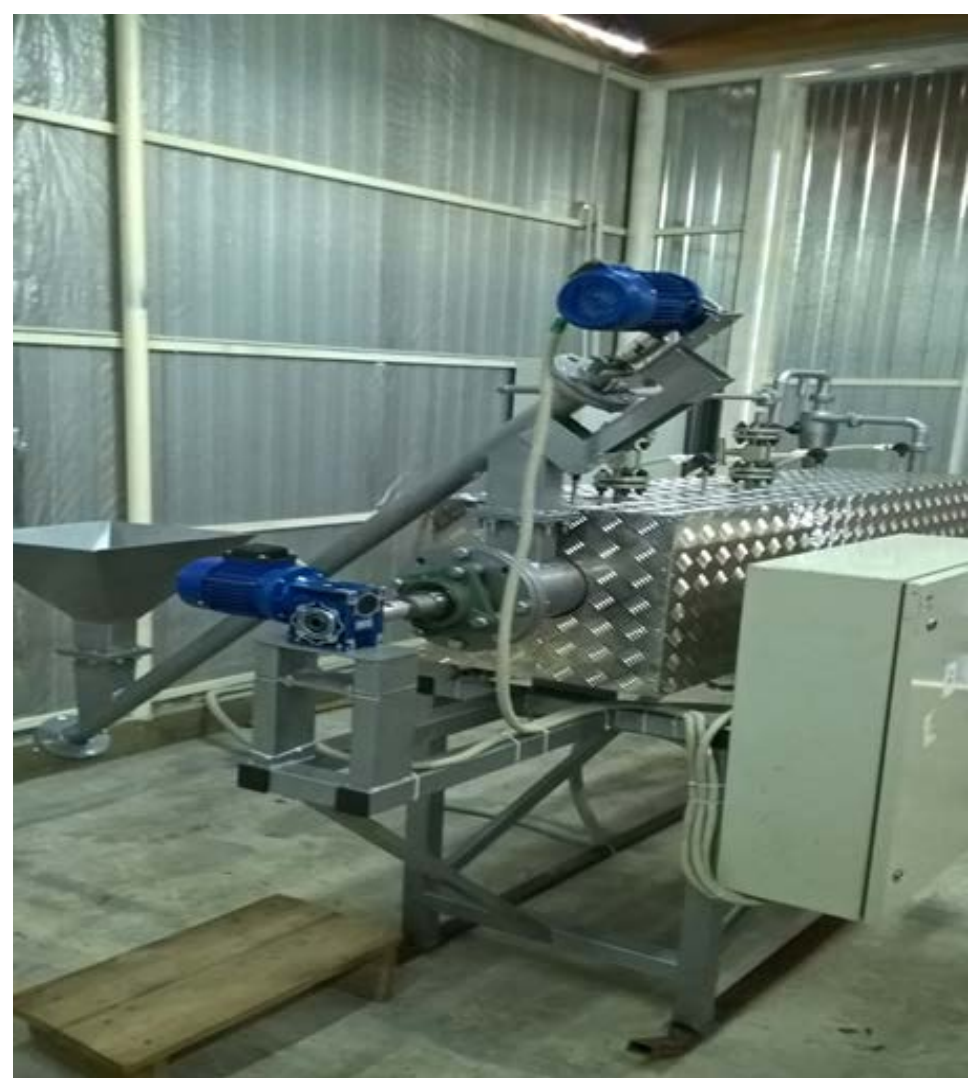

Figure 13. A pyrolysis reactor for solid biomass. 
quality. It is produced with high energy efficiency with respect to consumption of resources for reactor's heating. Yet we cannot disclose parameters of its exploitation due to a commercial character of its use. However, it is easy to conclude for the discussion, that mixing and transfer of biomass by an Archimedes screw makes possible phase changes of the alloy between the steel plates. One may have higher melting point of the alloy if proportion of the tin and bismuth is changed. It is obvious to assume, that the alloy inside this reactor possesses a higher melting point than the alloy applied for a direct contact with the hydrocarbons in our experiments. Because of this, the random and rapid releases of solidification heat of the alloy take place via the steel plates at as low as possible temperature for processing of solid biomass. Therefore, the above discussed hypothetical mechanism of an indirect energy transfer to the bonds of hydrocarbons in biomass may take place.

Furthermore, if one takes to account the above mentioned mechanism of realization of a Le Chatelier principle, one may argue, that application of the phase changing alloy as a heat conductor may improve efficiency of catalysts, whose work is based on a spatial ordering of micro-motions of reacting atoms and molecules. Such ordering has to be in the direction opposite to the direction of spreading of the sharp heat front. It's easy to conclude, that such ordering gives more time to atoms and molecules to react, when they move in the same direction. The rapid release of a solidification heat creates the best conditions for a highest magnitude of the ordering quantum like force to be obtained by Equation (1).

Having such argumentation in mind, a reactor, whose photo is presented in Figure 14 was designed and deployed to commercial exploitation. This reactor processes a pyrolysis oil to the high octane petrol (octane number equals to 104)

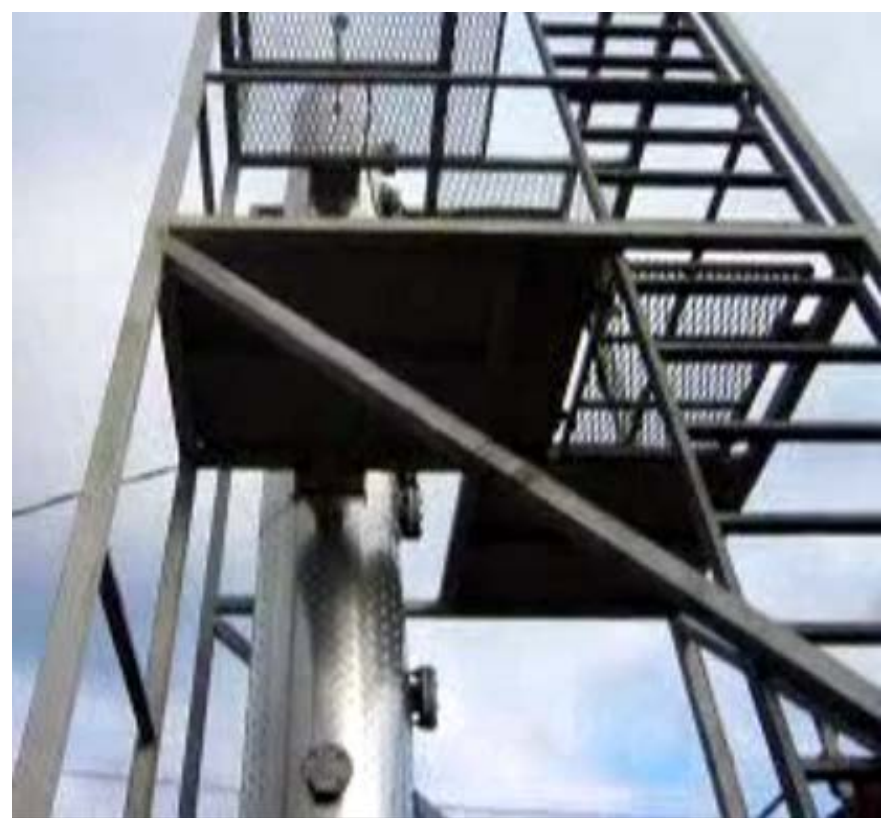

Figure 14. Reactor to process pyrolysis oil to a high octane petrol. 
using an ion exchanging catalyst, which is placed in a direct contact with the wall of an internal chamber having next the alloy, next a wall, which is heated. In a small prototype of this reactor, which is designated as a paraffines' isomerisation reactor, the heater was electrical. The industrial scale reactor has combustion as a source of heat to produce the high octane petrol from pyrolysis oil. Again, one of co-authors of this work and [4] inventor of the method to heat biomass through the phase-changing alloy, Oleksandr Ivanovych Vyhoniailo, is not allowed to disclose more technical details of construction of reactor on Figure 14. What is possible to say about application of the alloy in this reactor is that it does improve efficiency of the ion-exchange catalyst being applied in contact with the evaporated olefins of pyrolysis oil.

Another important note, which concerns efficiency of hydrocarbons decomposition in our experiments versus efficiency of this in reactors on Figure 13 and Figure 14 is that thickness of the alloy between the steel plates has to be not less than $7 \mathrm{~mm}$ for its efficient work. That was determined experimentally by authors of [4] and designers of reactors on Figure 13 and Figure 14. The thickness of the alloy in our experiments was in the range of 1 to $4 \mathrm{~mm}$. Along with the fact that the alloy was eutectic (it implies lowest magnitude of the alloy solidification temperature), this may explain, why the amount of hydrogen being obtained during the experiments was so low.

\section{Conclusions}

The focus of this paper is the determination of physical parameters, conditions and mechanisms of the low temperature pyrolysis of gaseous hydrocarbons that takes place due to their motion along hot fractal interfaces. The main conclusions may be summarized as follows.

1) The experiments on a low temperature mechanically assisted pyrolysis of a propane/butane mixture and biogas using several experimental setups showed a possibility to initiate a pyrolysis process at a gas temperature starting from $70^{\circ} \mathrm{C}$.

2) A mechanical assistance for the process was provided by the pulsating blowing of the gas, which moves along fractal surfaces that were created in the following three distinct ways:

a) By an under-cooling of the alloys having melting temperatures between $138^{\circ} \mathrm{C}$ and $140^{\circ} \mathrm{C}$.

b) By a mechanical fracturing of wires of the $\mathrm{Sn}$ :Bi alloy.

c) By a mechanical fracturing of the titanium dioxide films.

3) It was not possible to determine the exact physical mechanism of the low temperature cracking of hydrocarbons being observed in the experiments.

4) Two probable mechanisms of the energy transfer to vibrations of the bonds of the hydrocarbons were discussed. The first hypothetical mechanism of the energy transfer to the bonds being considered as oscillators in the field of the dynamic gradients was offered for a consideration by the corresponding author of the paper [14] in application to the high temperature pyrolysis of big wood 
samples. This hypothetical mechanism receives partial experimental support in the experiments described in this paper.

5) An alternative mechanism assumes a catalytic mechanism that promotes a low temperature cracking of hydrocarbons. This mechanism may work due to a rapid cooling of the Sn:Bi alloy by the gas, whose strong blowing also detaches the small metallic particles from the surface of the alloy. The particles may promote a catalytic decomposition of hydrocarbons during their motion in a closed loop of the experimental setup.

6) The proof or rejection of the later or the first mechanism requires more thorough measurements of the composition of particles to be found in the loop. Furthermore, more exact measurements of parameters such as the temperature, pressure and velocity of hydrocarbons during their motion along fractal interfaces are required. This is a topic of a future study.

\section{Acknowledgements}

The authors would like to express their gratitude for the Sintos Systems OÜ, SEM Laboratory at the Material Science Department of ITM School at KTH, and Division of Energy and Furnace Technology at the same Department for the financing and support of this work.

\section{References}

[1] Chen, G., Sjostrom, K. and Bjornbom, E. (1992) Pyrolysis/Gasification of Wood in a Pressurized Fluidized Bed Reactor. Industrial \& Engineering Chemistry Research, 31, 2164-2168. https://doi.org/10.1021/ie00012a021

[2] Gutfraind, R. and Sheintuch, S. (1992) Scaling Approach to Study Diffusion and Reaction Processes on Fractal Catalysts. Chemical Engineering Science, 47, 4425-4433. https://doi.org/10.1016/0009-2509(92)85120-Z

[3] Kantarelis, E., Yang, W. and Blasiak, W. (2013) Effects of Silica-Supported Nickel and Vanadium on Liquid Products of Catalytic Steam Pyrolysis of Biomass. Energy \& Fuels, 28, 591-599. https://doi.org/10.1021/ef401939g

[4] Shostak, T.A. and Vyhoniailo, O.I. (2007) Process for the Preparation of Synthesis Gas from Fossil Coal, EPA.

http://v3.espacenet.com/origdoc?DB=EPODOC\&IDX=UA79575\&F=0\&QPN=UA7 $\underline{9575}$

[5] Imre, A., Pajkossy, T. and Nyikos, L. (1992) Electrochemical Determination of the Fractal Dimension of Fractured Surfaces. Acta Metallurgica et Materialia, 40, 1819-1826. https://doi.org/10.1016/0956-7151(92)90168-E

[6] De Castro, W.B. (2005) Undercooling of Eutetic Sn-57wt\% Bi Alloy. Materials Science Forum, 480-481, 201-206. https://doi.org/10.4028/www.scientific.net/MSF.480-481.201

[7] Weimer, A.W., Dahl, J., Tamburini, J., Lewandowski, A., Pitts, R., Bingham, C. and Glatzmaier, G.C. (2000) Thermal Dissociation of Methane Using a Solar Coupled Aerosol Flow Reactor. Proceedings of the 2000 DOE Hydrogen Program Review NREL/CP-570-28890, 1-23.

[8] Mudry, S., Shtablavyi, I. and Shevernoga, I. (2013) Structural Disordering in $\mathrm{Sn}-\mathrm{Pb}(\mathrm{Bi})$ Eutectic Melts Induced by Heating. Polish Journal of Chemical Technol- 
ogy, 15, 61-64. https://doi.org/10.2478/pjct-2013-0045

[9] Sanyal, D., Ramachandrarao, P. and Gupta, O.P. (2005) A Fractal Description of Transport Phenomena in Dendritic Porous Network. Chemical Engineering Science, 61, 307-315. https://doi.org/10.1016/j.ces.2005.06.005

[10] Nottale, L. (2008) Scale Relativity and Fractal Space-Time: Theory and Applications. Proceedings of 1 st International Conference on the Evolution and Development of the Universe, Paris, Vol. 15, 101-152.

[11] Notalle, L. (2009) Generalized Quantum Potentials. Journal of Physics A: Mathematical and Theoretical, 42, 275-306. https://doi.org/10.1088/1751-8113/42/27/275306

[12] Nottale, L. and Lehner, T. (2012) Numerical Simulation of a Macroscopic Quantum-Like Experiment: Oscillating Wave Packet. International Journal of Modern Physics C, 23, 1-27. https://doi.org/10.1142/S0129183112500350

[13] Moon, K.W., Boettinger, W.J., Kattner, U.R., Handwerker, C.A. and Lee, D.J. (2001) The Effect of $\mathrm{Pb}$ Contamination on the Solidification Behavior of Sn-Bi Solders. Journal of Electronic Materials, 30, 45-52.

https://doi.org/10.1007/s11664-001-0213-x

[14] Alevanau, A., Yang, W., Blasiak, W., Kuznechik, O.P. and Vyhoniailo, O.I. (2013) Prospective Side Effects of the Heat and Mass Transfers in Micro-Porous Structure of Char during Intermediate and Final Stages of the High-Temperature Pyrolysis. Nonlinear Phenomena in Complex Systems, 16, 287-301. 